\title{
Screening Donated Blood for Transfusion Transmitted Infections by Serology along with NAT and Response Rate to Notification of Reactive Results: An Indian Experience
}

\author{
Rahul Chaurasia, Shamsuz Zaman, Bankim Das, and Kabita Chatterjee \\ Department of Transfusion Medicine, All India Institute of Medical Sciences, New Delhi 110029, India \\ Correspondence should be addressed to Shamsuz Zaman; rxhope@gmail.com
}

Received 13 August 2014; Revised 15 September 2014; Accepted 28 October 2014; Published 16 November 2014

Academic Editor: Silvano Wendel

Copyright (C) 2014 Rahul Chaurasia et al. This is an open access article distributed under the Creative Commons Attribution License, which permits unrestricted use, distribution, and reproduction in any medium, provided the original work is properly cited.

Background. Transfusion safety begins with healthy donors. A fundamental part of preventing transfusion transmitted infections (TTIs) is to notify and counsel reactive donors. Donor notification and counselling protect the health of the donor and prevent secondary transmission of infectious diseases. Methods. 113,014 donations were screened for TTIs, namely, HIV, HBV, HCV, and syphilis, by serology and nucleic acid testing. All reactive donors were retested (wherever possible) and notified of their status by telephone or letter. All initial reactive screens were followed over six months. Results. We evaluated 2,838 (2.51\%) cases with reactive screening test results $(1.38 \% \mathrm{HBV}, 0.54 \% \mathrm{HCV}, 0.27 \% \mathrm{HIV}$, and $0.32 \%$ syphilis). Only $23.3 \%$ of donors (662) responded to notification. The response among voluntary donors was better as compared to the replacement donors (43.6\% versus $21.2 \%)$. Only $373(56.3 \%)$ responsive donors followed their first attendance at referral specialties. Over six months, only 176 of $662(26.6 \%)$ reactive donors received treatment. Conclusion. Our study shed light on the importance of proper donor counselling and notification of TTI status to all reactive donors who opt to receive this information. There is also an urgent need to formulate the nationally acceptable guidelines for notification and follow-up of reactive donors.

\section{Introduction}

Blood transfusion is safer than ever before through continuous improvements in donor recruitment, screening, testing of donated blood with increasingly sensitive assays, and appropriate clinical use of blood [1]. Serologic testing for transfusion transmitted diseases had historically been the foundation of blood screening, while newer strategies like nucleic acid testing (NAT) have helped further shorten the "window period" [2]. Currently, no technology exists to completely detect all window period donations. No matter how sensitive NAT becomes, we will never be able to completely close the exposure-to-seroconversion window period. The general public and media might believe that with the advancement in testing technologies zero risk blood products are currently available. This generalization is far from reality as judged by our current experience with new testing methodologies. Breakthrough transmissions of viruses (HIV1 and HCV) had occurred as late as 2009 due to NAT failures because of low level of viraemia and/or suboptimal amplification efficiency [3]. Moreover, threat of infectious agents entering the blood supply is not static and may evolve as new pathogens emerge or as old ones change their epidemiological pattern [4]. Therefore, regardless of testing modality chosen, a nonzero risk of disease transmission still exists in all its seriousness [5]. Under current practice in India, potential blood donors, after registration, filling of donor-health questionnaire, and brief medical examination, are sent for predonation counseling. During counseling process of blood donation, postdonation care and the outcomes of donation are explained. After blood donation, samples are collected for screening for anti-HIV-1/2, anti-HCV, and HBsAg, RPR for syphilis, and slide/card test for malaria. In addition to ELISA, NAT is being increasingly used in many centers to further 
improve blood safety although it is not mandated by national authorities.

In 2002, the Government of India adopted the National Blood Policy "An action plan for blood safety" to ensure safe blood supply. This policy advocates notification to all reactive blood donors. Blood banks are thus now required to obtain written consent from donors at time of donation for screening blood for TTI (Transfusion-transmitted Infections) and whether they wish to be informed about their abnormal tests results [6]. If any of the screening tests are abnormal, before notification to the donors the tests are repeated either using two assays of differing principles or in duplicate with the same assay so as to avoid notification of false-positive results. Donors who report back to the transfusion facility are retested and if found repeat reactive are referred to integrated counseling and testing centers (ICTC) for HIV and gastroenterology and STD clinic for $\mathrm{HBV} / \mathrm{HCV}$ and syphilis, respectively, for counseling, confirmatory testing, and management.

Currently in India, most of the blood banks including ours do not have the facility to perform confirmatory tests for TTI. The donors are informed only on the basis of their screening tests available in blood bank. As most of the donors do not expect to hear that they have reactive results they may become extremely distressed to hear this news. These donors may be highly motivated to donate, having desire to help others, or simply want some time off work or may have other motives. This, unfortunately, may leave the donor with a negative feeling towards blood donation or diminish his/her own self-worth $[7,8]$. On the other hand, a small minority of individuals appear to ignore notification and continue to donate blood elsewhere. Some of the donors even use blood donations as a means for free testing because of their high risk behavior (test seekers) [9].

Donor notification can therefore be a challenging task demanding special skills from the staff involved who should always be prepared to meet new challenges and help donors come to terms with their newly discovered status. Although the blood policy advocates disclosure of TTI status, donors are not, in practice, informed about their results. The onus lies with the donor to contact the blood bank [10]. There is very little information available about donor behavior on receipt of reactive TTI results. We undertook this study to determine the response rate following notification of reactive status to the donors. We also assessed the prevalence of TTI using serology as well as NAT among blood donors attending our center.

\section{Material and Methods}

Between January 2011 and December 2013, 113,014 donations at Main Blood Bank, All India Institute of Medical Sciences, New Delhi, were screened by ELISA using 4th generation ELISA test kits for HIV-1/2, 3rd generation ELISA test for HBsAg and $\mathrm{HCV}$ infections, and rapid immunochromatographic test for syphilis. All donations were also screened individually using the Procleix Ultrio assay (Novartis, Emeryville, CA), a multiplex NAT assay for the detection of hepatitis B virus (HBV) DNA, hepatitis $\mathrm{C}$ virus (HCV) RNA, and human immunodeficiency virus1 (HIV-1) RNA. If initial NAT result was positive, sample was retested again in triplicate. If any of these three tests were positive, sample was drawn from plasma bag and tested in triplicate. Discriminatory NAT was run if any test from plasma bag came positive. The analytical sensitivities of the Procleix Ultrio assay for HBV DNA, HCV RNA, and HIV-1 RNA are $10.4(9.2-12.2) \mathrm{IU} / \mathrm{mL}, 3.0$ (2.7-3.4) IU/mL, and 47.9 (43.3-54.5) IU/mL, respectively. The analytical sensitivities for the Procleix dHBV ( $\mathrm{d}=$ discriminatory), $\mathrm{dHCV}$, and dHIV assays are $8.5(7.6-9.8) \mathrm{IU} / \mathrm{mL}, 3.2(2.8-3.6) \mathrm{IU} / \mathrm{mL}$, and 53.6 (47.9-61.2) IU/mL, respectively.

If the results of either serology and/or NAT were found to be positive, blood unit was discarded as per hospital SOPs and donor was notified of his/her status either by telephone or by letter. The first follow-up call was made on the 10th day of notification. If the donor did not respond to this first call, second and third follow-up calls were made every 10 days. The case was closed only if the donor did not respond to any of the three telephone calls/letters and the case was labeled as nonresponder. Donors who responded to the call/letters and came back to transfusion facility were counseled and retested by ELISA with fresh blood sample. Donors whose results from fresh sample were concordant with earlier tests were referred to concerned clinical specialty and donors who tested nonreactive were asked to remain in follow-up (Figure 1).

\section{Results}

A total of 113,014 donors were evaluated comprising $85.4 \%$ replacement and $14.6 \%$ voluntary donors. The majority of the donors (97\%) donated blood for the first time. The demographic details of donors are given in Table 1. Total of $2838(2.51 \%)$ donors tested reactive for TTI. Prevalence of TTI was $1.38 \%$ for HBV, $0.54 \%$ for HCV, $0.27 \%$ for HIV, and $0.32 \%$ for syphilis (Table 2). For HIV, HBV, and HCV testing, concordant serological and NAT reactive results were found in 1643/2480 (66.25\%) positive donations. NAT yield in our study was 1 in 628 donations (180 NAT+ELISAcases) (Table 2). Of all the donors who were notified of their reactive status only $662(23.3 \%)$ donors reported back to transfusion facility. The response among voluntary donors was better as compared to the replacement donors $(43.6 \%$ versus 21.2\%) (Table 3). Donors residing in the urban nearby areas responded better than those who lived in rural or far-off areas. Donor notification using telephone was more beneficial as more donors turned up to transfusion facility. Only 373 (56.3\%) responsive donors followed their first attendance at referral specialties. Over six months, 176 of 662 (26.6\%) responsive donors were undergoing treatment.

\section{Discussion}

With over 93 million donations made every year worldwide, blood transfusion continues to save millions of lives each year and improve the life expectancy and quality of life of patients 


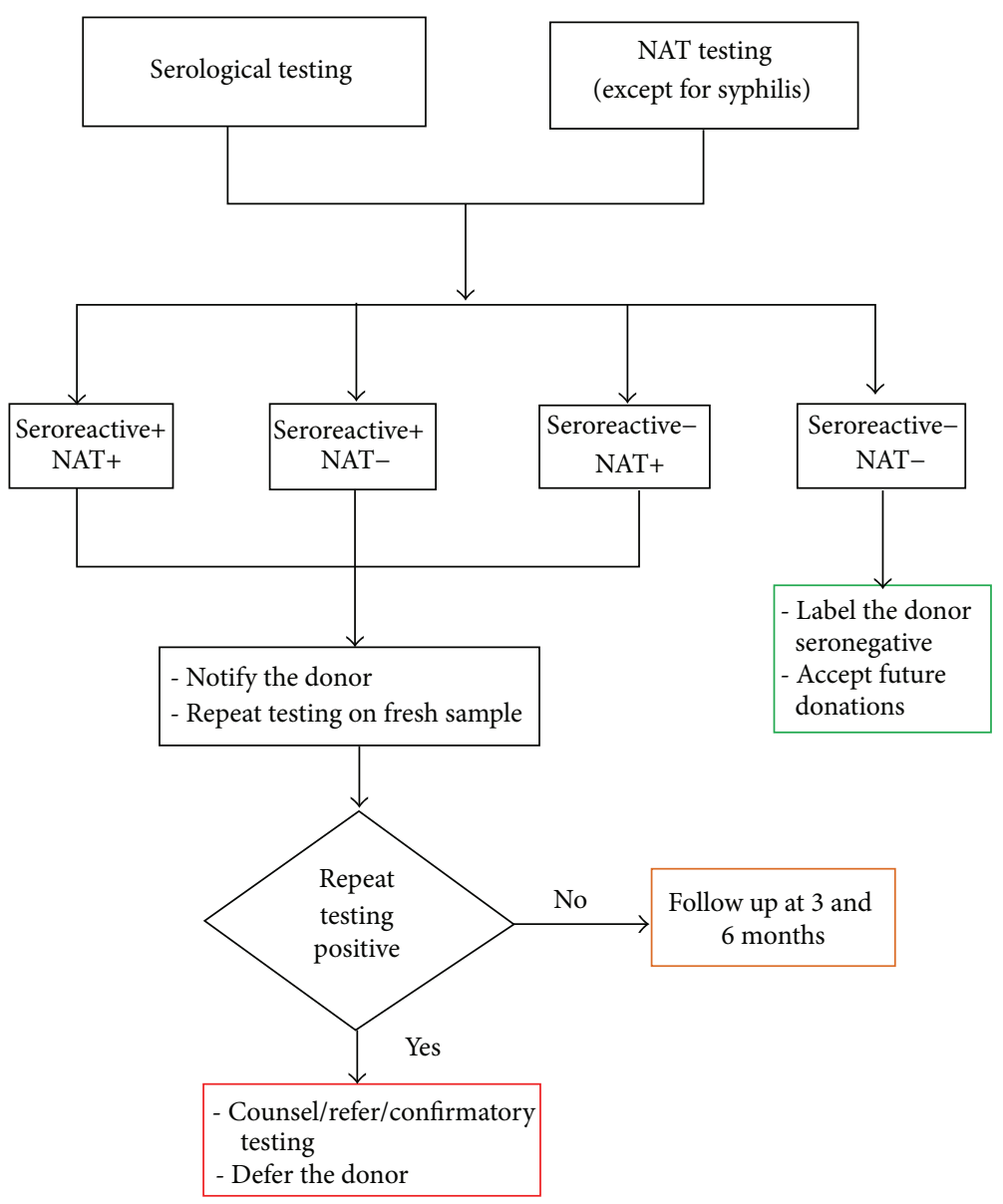

FIGURE 1: Algorithm for donor testing and recall.

TABLE 1: Demographic details of donations.

\begin{tabular}{lcc}
\hline \multicolumn{2}{c}{ Donor demographics $(n=113,014)$} & $\%$ \\
\hline Gender & Number & \\
Male & 108,042 & 95.6 \\
Female & 4,972 & 4.4 \\
Age group & & \\
18-25 & 36,390 & 32.2 \\
$26-40$ & 61,367 & 54.3 \\
41-65 & 15,257 & 13.5 \\
Residence & & \\
Urban & 78,206 & 69.2 \\
Rural & 34,808 & 30.8 \\
Donation type & & \\
Voluntary & 16,516 & 14.6 \\
Replacement & 96,498 & 85.4 \\
Donor repeatability & & 97 \\
First time donors & 109,669 & 3 \\
Repeat donor & 3,345 &
\end{tabular}

suffering from life-threatening conditions [11]. At the same time, blood transfusion is an important mode of transmission of infection to the recipients. Prevalence of TTI in India is $1.8-4 \%, 0.4-1.09 \%, 0.2-1 \%$, and $0.05-0.9 \%$ for $\mathrm{HBV}, \mathrm{HCV}$, HIV, and syphilis, respectively [12-17]. Prevalence of TTI in the present study was in agreement with other seroprevalence studies carried out in various parts of India. NAT yield in our study was 1 in 628 donations which was comparable to a previous study performed at the same institution [18]. There was high proportion of ELISA positive/NAT negative for HIV (50\%) and HBV (15\%) in our study (Table 2 ). This could be either due to low viral load below detection limits of the NAT assay or due to false-positive results in ELISA. The differences in the proportion of ELISA positive/NAT negative for HIV (50\%) and HBV (15\%) in our study can be explained by the fact that we performed fourth generation ELISA testing for $\mathrm{HIV}$ which has a high potential risk for nonspecific reactivity [19].

Transfusion safety begins with healthy donors. A fundamental part of preventing TTI is to notify and counsel reactive donors. Donor notification and counseling protect the health of the donor, prevent secondary transmission of infectious diseases to sexual partners, reduces risk of vertical transmission and provide feedback about the effectiveness of donor selection procedures such as predonation education and medical history [20]. We attempted to contact all 
TABLE 2: Prevalence of TTI markers and comparison of ELISA versus NAT.

\begin{tabular}{|c|c|c|c|c|c|}
\hline Marker & Reactive donors & $\begin{array}{c}\text { ELISA+/NAT } \\
(\%)\end{array}$ & $\begin{array}{c}\text { ELISA+/NAT+ } \\
(\%)\end{array}$ & $\begin{array}{c}\text { ELISA-/NAT+ } \\
(\%)\end{array}$ & $\begin{array}{c}\text { Prevalence } \\
(\%)\end{array}$ \\
\hline $\mathrm{HBV}$ & 1557 & $232(0.21)$ & $1218(1.08)$ & $107(0.09)$ & 1.38 \\
\hline $\mathrm{HCV}$ & 612 & $269(0.24)$ & $272(0.24)$ & $71(0.06)$ & 0.54 \\
\hline HIV & 311 & $156(0.14)$ & $153(0.13)$ & $2(0.002)$ & 0.27 \\
\hline Syphilis (RPR) & 358 & NA & NA & NA & 0.32 \\
\hline Total & 2838 & 657 & 1643 & 180 & 2.51 \\
\hline
\end{tabular}

${ }^{*}$ NA: not applicable.

TABLE 3: Donor response rate.

\begin{tabular}{|c|c|c|c|c|c|c|c|}
\hline \multirow{2}{*}{ Marker } & \multirow{2}{*}{ Reactive donors } & \multicolumn{3}{|c|}{ Voluntary donors } & \multicolumn{3}{|c|}{ Replacement donors } \\
\hline & & Notified & Response & $\%$ & Notified & Response & $\%$ \\
\hline HBV & 1557 & 143 & 69 & 48.2 & 1414 & 244 & 17.3 \\
\hline $\mathrm{HCV}$ & 612 & 75 & 42 & 56 & 537 & 140 & 26.1 \\
\hline HIV & 311 & 39 & 5 & 12.8 & 272 & 91 & 33.5 \\
\hline Syphilis & 358 & 18 & 4 & 22.2 & 340 & 67 & 19.7 \\
\hline Total & 2838 & 275 & 120 & 43.6 & 2563 & 542 & 21.2 \\
\hline
\end{tabular}

$2838(2.51 \%)$ reactive donors about their TTI status either telephonically or by letter. Only $662(23.3 \%)$ reactive donors responded to the notification. In an Indian study by Patel et al. $236(60.36 \%)$ donors showed a positive response following donor notification [6]. In another study by Agarwal et al. involving 416 reactive donors, only 249 (59.8\%) donors turned to transfusion facility and attended counseling after receipt of their reactive status [21]. The counseling success rate at large blood center in southern India was $41.18 \%, 11.11 \%$, and $14.63 \%$ for HBV, HIV, and HCV, respectively [22]. Donor response rate in our study was low as compared to other studies from the country. This may be due to poor health care knowledge, social stigma associated with TTIs (especially HIV), and inadequate understanding of implications of screening tests among the general population [21, 23]. Also, as our center is a large volume referral center in India, most of the donors belonged to far-off places; thus distance could be a reason for the donors not reporting back to transfusion facility. Given the large volume of daily donations coupled with limited resources (only one counselor), it is difficult to ensure that every donor had understood the meaning and intent of counseling to the best of his/her intelligence. Another explanation which we think of for low response rate was nonreceipt of postal letters as donors tend to write inadequate postal address in donor registration forms. The study by Kleinman et al. reports that following notification $27 \%$ of donors contacted the blood center for further information [24].

One more finding of this study that should be a serious concern for blood transfusion authorities is that only 373 (56.3\%) of 662 responsive donors responded to the first call and followed up their attendance at the ICTC or with the physicians they were asked to meet. Rest of the 289 donors $(43.7 \%)$ was lost to follow up at this very first stage. This raises questions about the way donors are counseled and made aware of the consequences of not taking proper treatment. It is also alarming that only 176 donors $(6.2 \%)$ of all the reactive donors either were taking treatment or had completed it 6 months after having received notification of their infectious status. We could not find any studies from India to compare this data. The donors who did not turn up to transfusion facility (nonresponders) may continue to donate blood at other centers especially those centers which do not use biometric donor identification, hence posing serious threat to safety of blood supply. This threat is amplified by the fact that though ELISA is the recommended and preferred screening technique, many blood centers still do not have this facility and rely on "rapid kits" which may have high falsenegative rate. Donors who are ELISA/NAT reactive elsewhere may escape TTI screening [25].

As per objective 4.16 of the Indian action plan for blood safety, the blood donors are counseled about TTIs prior to donation and are offered the option of knowing their seroreactive status provided they give their consent. Low donor response rate suggest that we are not able to meet this goal with reasonable satisfaction. Another important problem which we encounter in our daily transfusion practice is about donors who are notified because of NAT reactive/ELISA nonreactive status. These donors have their tests repeated by their personal physicians and return with discrepant results as most of the laboratories again screen samples by ELISA. These donors usually demonstrate an angry behaviour in blood bank and question the accuracy of screening performed in blood bank. More often than not despite our best attempts we are not able to explain the meaning of sensitivity and specificity of testing methods to the donors up to their level of understanding. We need to follow up such cases over 6 months as $95 \%$ of infected persons will seroconvert in this timeframe [26].

Our study has two limitations. First, we did not perform confirmatory testing of TTIs prior to notification. Second, repeat NAT was not done on the returning reactive donors 
(although a quantitative viral load is being performed at the referral center, unfortunately the results of which are not available to us). Our study was a small endeavor in determining donor behavior when informed about their reactive status according to results based on screening assays including NAT. Transfusion safety rests heavily on the health of blood donors. To improve donor response rate, we have switched to exclusive telephonic notification to all donors who test reactive in screening tests. Donors should undergo optimal predonation counseling so as to educate them about the risk of infections and the window period. It is the collective duty of transfusion community to inform these donors and do as much as possible to allay their anxiety about reactive result and to advise them about available treatment options. There is an urgent need to formulate the nationally acceptable guidelines for notification of all reactive donors. We have tried to formulate such schema (Figure 1). We expect that other blood centers will carry out such studies and further refine the algorithm.

\section{Conflict of Interests}

The authors declare that there is no conflict of interests regarding the publication of this paper.

\section{References}

[1] Z. S. Bharucha, "Safe blood transfusion practices," Indian Journal of Pediatrics, vol. 68, no. 2, pp. 127-131, 2001.

[2] R. Hans and N. Marwaha, "Nucleic acid testing-benefits and constraints," Asian Journal of Transfusion Science, vol. 8, no. 1, pp. 2-3, 2014.

[3] C. M. Nübling, M. Heiden, M. Chudy et al., "Experience of mandatory nucleic acid test (NAT) screening across all blood organizations in Germany: NAT yield versus breakthrough transmissions," Transfusion, vol. 49, no. 9, pp. 1850-1858, 2009.

[4] F. Bihl, D. Castelli, F. Marincola, R. Y. Dodd, and C. Brander, "Transfusion-transmitted infections," Journal of Translational Medicine, vol. 5, article 25, 2007.

[5] L. M. Kucirka, H. Sarathy, P. Govindan et al., "Risk of window period HIV infection in high infectious risk donors: systematic review and meta-analysis," The American Journal of Transplantation, vol. 11, no. 6, pp. 1176-1187, 2011.

[6] D. P. Patel, D. S. Patel, D. J. Bhatt, D. N. Bhatnagar, and D. M. G. Shah, "Evaluation of response to donor notification of reactive transfusion transmitted infections (ttis) result," National Journal of Integrated Research in Medicine, vol. 3, no. 2, pp. 20-25, 2012.

[7] L. A. Bashawri, "A review of predonation blood donor deferrals in a university hospital," Journal of Family and Community Medicine, vol. 12, no. 2, pp. 79-84, 2005.

[8] K. A. Munsterman, A. J. Grindon, M. T. Sullivan et al., "Assessment of motivations for return donation among deferred blood donors," Transfusion, vol. 38, no. 1, pp. 45-50, 1998.

[9] T. M. Roshan, H. Rosline, S. A. Ahmed, M. Rapiaah, and M. N. Khattak, "Response rate of malaysian blood donors with reactive screening test to transfusion medicine unit calls," The Southeast Asian Journal of Tropical Medicine and Public Health, vol. 40, no. 6, pp. 1315-1321, 2009.
[10] L. P. Choudhury and S. Tetali, "Ethical challenges in voluntary blood donation in Kerala, India," Journal of Medical Ethics, vol. 33, no. 3, pp. 140-142, 2007.

[11] Global blood safety and availability. Key facts and figures, 2010.

[12] P. Kaur and S. Basu, "Transfusion-transmitted infections: existing and emerging pathogens," Journal of Postgraduate Medicine, vol. 51, no. 2, pp. 146-151, 2005.

[13] D. Arora, B. Arora, and A. Khetarpal, "Seroprevalence of HIV, HBV, HCV and syphilis in blood donors in Southern Haryana," Indian Journal of Pathology and Microbiology, vol. 53, no. 2, pp. 308-309, 2010.

[14] P. Pallavi, C. K. Ganesh, K. Jayashree, and G. V. Manjunath, "Seroprevalence and trends in transfusion transmitted infections among blood donors in a University Hospital blood bank: a 5 year study," Indian Journal of Hematology and Blood Transfusion, vol. 27, no. 1, pp. 1-6, 2011.

[15] K. Chatterjee, P. Coshic, M. Borgohain, R. M. Thapliyal, S. Chakroborty, and S. Sunder, "Individual donor nucleic acid testing for blood safety against HIV-1 and hepatitis b and c viruses in a tertiary care hospital," National Medical Journal of India, vol. 25, no. 4, pp. 207-209, 2012.

[16] T. Chandra, S. N. F. Rizvi, and D. Agarwal, "Decreasing prevalence of transfusion transmitted infection in Indian scenario," The Scientific World Journal, vol. 2014, Article ID 173939, 4 pages, 2014.

[17] M. Meena, T. Jindal, and A. Hazarika, "Prevalence of hepatitis $B$ virus and hepatitis $C$ virus among blood donors at a tertiary care hospital in India: a five-year study," Transfusion, vol. 51, no. 1, pp. 198-202, 2011.

[18] N. Agarwal, K. Chatterjee, P. Coshic, and M. Borgohain, "Nucleic acid testing for blood banks: an experience from a tertiary care centre in New Delhi, India," Transfusion and Apheresis Science, vol. 49, no. 3, pp. 482-484, 2013.

[19] L. Gürtler, A. Mühlbacher, U. Michl et al., "Reduction of the diagnostic window with a new combined p24 antigen and human immunodeficiency virus antibody screening assay," Journal of Virological Methods, vol. 75, no. 1, pp. 27-38, 1998.

[20] C. Bianco and D. Kessler, "Donor notification and counseling management of blood donors with positive test results," Vox Sanguinis, vol. 67, no. 3, pp. 255-259, 1994.

[21] N. Agarwal, "Response rate of blood donors in the uttarakhand region of india after notification of reactive test results on their blood samples," Blood Transfusion, vol. 12, no. 1, pp. s51-s53, 2014.

[22] S. Dontula, A. Mathur, T. Kamaladoss, S. Adimurthy, and L. Jagannathan, "Donor disclosure-a donor's right and blood bank's responsibility," Transfusion Alternatives in Transfusion Medicine, vol. 12, no. 3-4, pp. 44-50, 2012.

[23] N. Choudhury, P. Singh, and H. Chandra, "AIDS awareness in blood donors in north India," Transfusion Medicine, vol. 5, no. 4, pp. 267-271, 1995.

[24] S. Kleinman, B. Wang, Y. Wu et al., "The donor notification process from the donor's perspective," Transfusion, vol. 44, no. 5, pp. 658-666, 2004. 
[25] V. P. Torane and J. S. Shastri, "Comparison of ELISA and rapid screening tests for the diagnosis of HIV, hepatitis B and hepatitis $\mathrm{C}$ among healthy blood donors in a tertiary care hospital in Mumbai," Indian Journal of Medical Microbiology, vol. 26, no. 3, pp. 284-285, 2008.

[26] C. A. Ciesielski and R. P. Metler, "Duration of time between exposure and seroconversion in healthcare workers with occupationally acquired infection with human immunodeficiency virus," The American Journal of Medicine, vol. 102, no. 5, pp. 115116, 1997. 


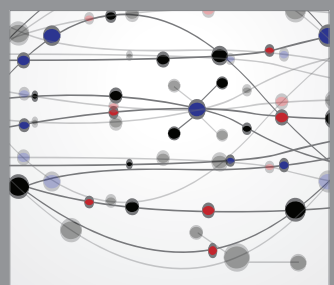

The Scientific World Journal
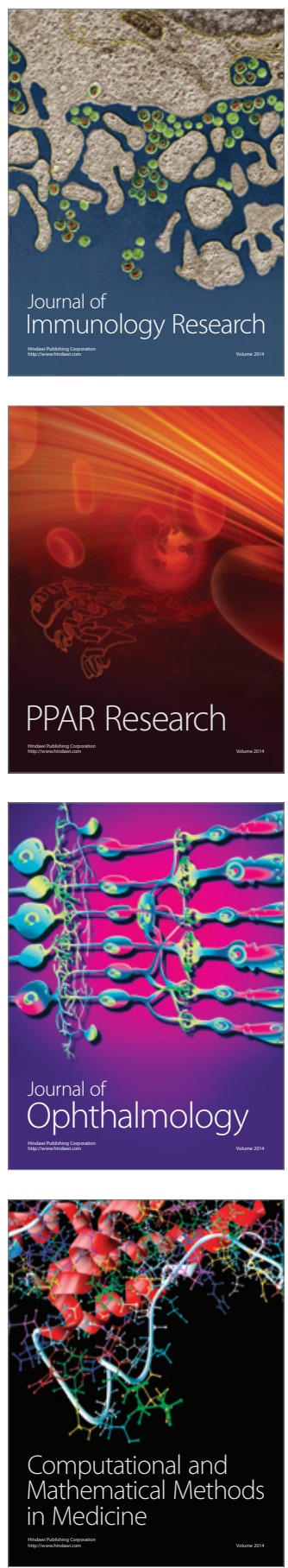

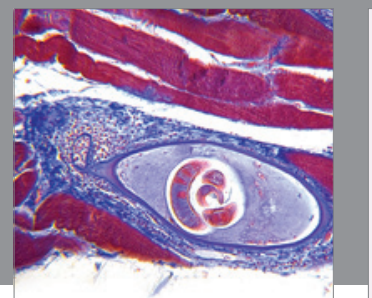

Gastroenterology

Research and Practice
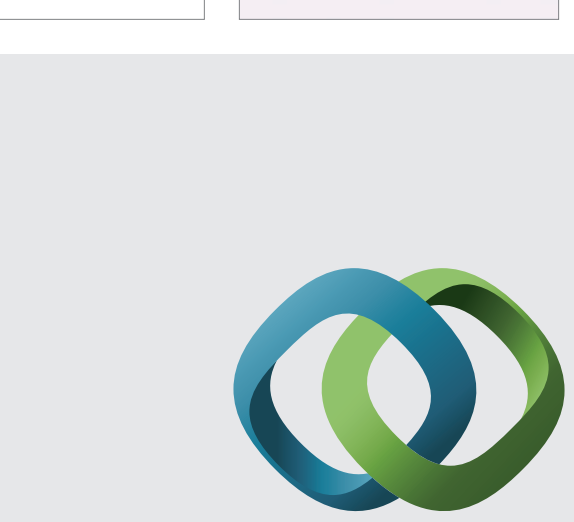

\section{Hindawi}

Submit your manuscripts at

http://www.hindawi.com
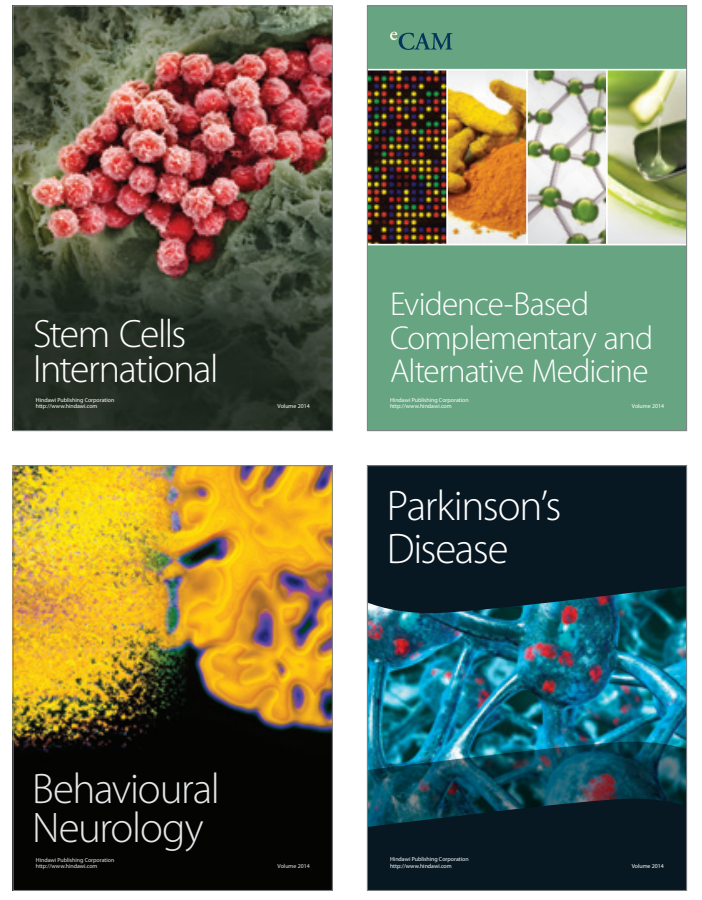
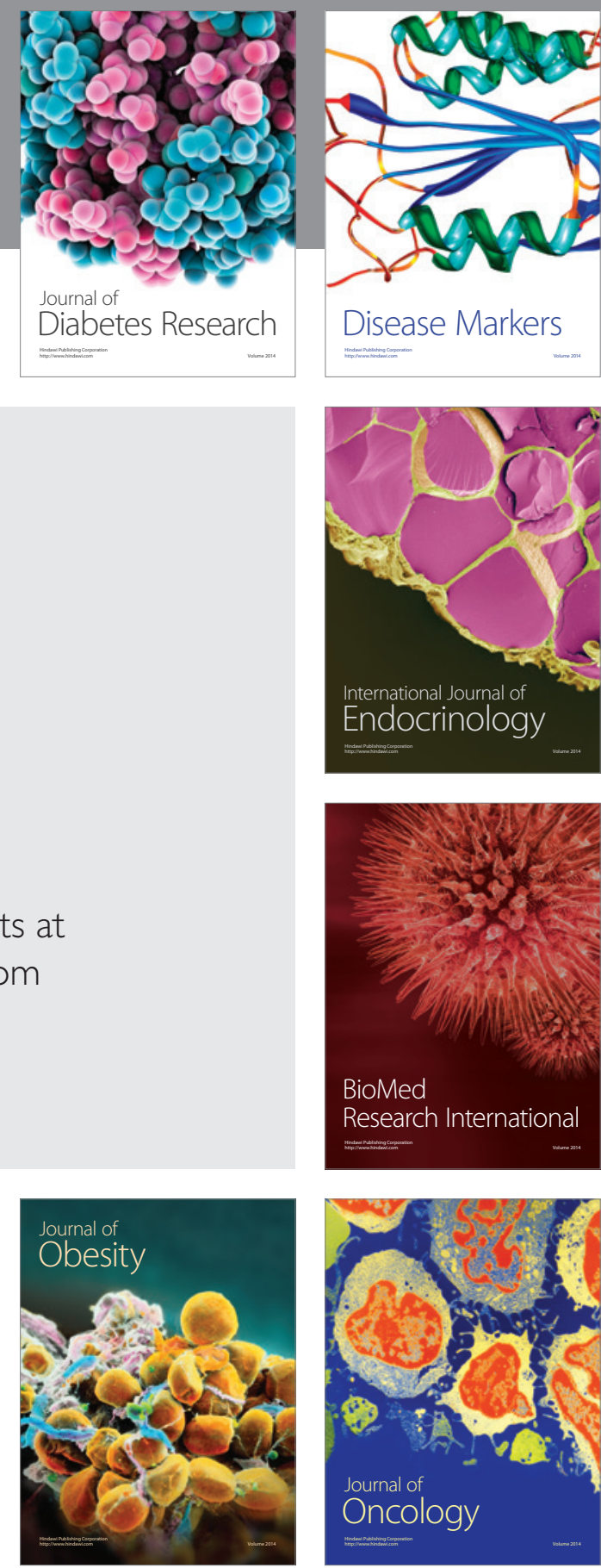

Disease Markers
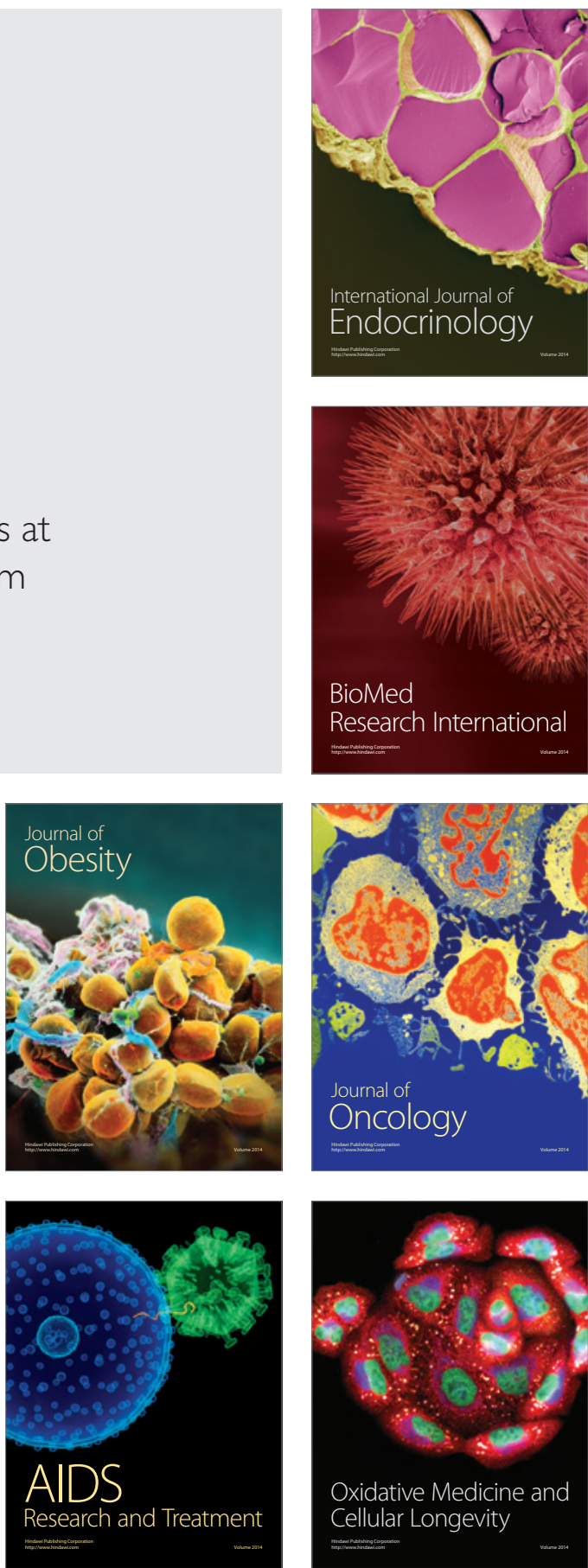